\title{
COMPETENCIA PERCIBIDA, PROCESOS DE VALORACIÓN Y AFRONTAMIENTO ANTE UN SUPUESTO PROBLEMA CARDIOVASCULAR
}

\author{
BEATRIZ RUEDA Y ANA M. PÉREZ-GARCÍA \\ Universidad Nacional de Educación a Distancia
}

(Aceptado en septiembre de 2005)

\begin{abstract}
Este estudio pretendió estudiar la contribución de los estilos de afrontamiento, la competencia percibida en salud (CPS) y las valoraciones primaria y secundaria sobre el afrontamiento situacional. Las relaciones entre la CPS y las valoraciones también se estudiaron. La muestra se compuso de 110 estudiantes, quienes indicaron sus reacciones ante una supuesta tensión arterial alta. Los estilos de afrontamiento se asociaron de forma moderada con el afrontamiento situacional. Junto con la CPS, explicaron un porcentaje de variabilidad de las estrategias centradas en el problema y la emoción positiva, similar al mostrado por las valoraciones. La contribución de las valoraciones fue superior en el afrontamiento emocional negativo. Asimismo, la CPS se asoció con la valoración primaria y secundaria. Estos datos sugieren que, tanto las valoraciones específicas, como la competencia percibida y los estilos de afrontamiento previos, son factores que determinan la forma de afrontar un problema de salud

Palabras clave: Competencia percibida, valoración, afrontamiento, enfermedad cardiovascular.

\section{Perceived competence, appraisal processes and coping in response to a supposed cardiovascular problem}

This study aimed at examining the contribution of the coping styles, perceived health competence (PHC) and primary and secondary appraisals on situational coping. Relations between PHC and appraisals were also studied. The sample was composed by 110 students, who reported their reactions to a supposed high blood pressure. The coping styles were moderately associated with situational coping. Together with PHC, they explained a similar percentage of the variability of situational problem-focused and positive emotion-focused coping to the one shown by the appraisals. The contribution of appraisals was higher concerning negative-emotion oriented coping. Furthermore, PHC was associated with primary and secondary appraisals. These data suggest that both, the specific appraisals, and the previous perceived competence and coping styles, are factors that determine the way to cope with a health problem
\end{abstract}

Key words: Perceived competence, appraisal, coping, cardiovascular disease.

\section{INTRODUCCIÓN}

El estudio de la forma como las personas perciben y reaccionan ante los acontecimientos estresantes ha sido objeto de una amplia investigación. Gran parte de estos trabajos se ha centrado en examinar el papel que desempeñan los recursos per-

Correspondencia: Beatriz Rueda, Facultad de Psicología (UNED), Departamento de Psicología de la Personalidad, Evaluación y Tratamiento Psicologicos, Juan del Rosal, 10, 28040 Madrid. Correo-e: brueda@psi.uned.es sonales, los procesos de valoración (Terry, 1994) y las estrategias de afrontamiento (Muela, Torres y Peláez, 2002), en la reducción del impacto subjetivo de las situaciones estresantes.

Uno de los marcos téricos, en los que más ampliamente se ha sustentado la investigación del afrontamiento, es el propuesto por Lazarus y Folkman (Folkman, Lazarus, Dunkel-Schetter, DeLongis, y Gruen, 1986). De acuerdo con estos autores, dos determinantes básicos en el 
manejo del estrés son los procesos de valoración y las estrategias de afrontamiento. Dentro de los procesos de valoración, la valoración primaria se refiere al significado que tiene un acontecimiento para el bienestar de la persona (valoración irrelevante, positiva o estresante). Así, un suceso se percibirá como estresante en la medida en que implique un cierto grado de reto, amenaza o daño. La valoración secundaria, por su parte, se basa en la estimación de la capacidad disponible para hacer frente a la situación de estrés.

De otro lado, las estrategias de afrontamiento representan el esfuerzo cognitivo, conductual y emocional que se realiza para alterar las características del estresor (afrontamiento centrado en el problema), o para regular las reacciones afectivas que ante él se desencadenan (afrontamiento centrado en la emoción). Si bien Lazarus y Folkman (1987) consideran las valoraciones y las estrategias de afrontamiento como procesos contextuales ligados a las situaciones donde se originan, su modelo también incluye ciertas características de personalidad más generales como posibles antecedentes de los procesos de valoración. Un aspecto importante de la consideración de estas variables es la posibilidad de dar cabida a los estilos de afrontamiento dentro del proceso de estrés, entendiendo por estilo o afrontamiento disposicional la tendencia a reaccionar ante los acontecimientos estresantes de forma estable y consistente (Endler y Parker, 1994).

Algunos estudios, como los realizados por Carver y Scheier (Carver y Scheier, 1994; Carver, Scheier y Weintraub, 1989) y Bouchard (Bouchard, Guillemette y Landry-Léger, 2004), han puesto de manifiesto que los estilos de afrontamiento $y$ sus correspondientes estrategias de afrontamiento se asocian de forma moderada aunque significativa, oscilando la magnitud de las correlaciones entre .23 y .39.
No obstante, otros trabajos reflejan una relación mucho más débil entre el afrontamiento disposicional y el situacional. Así, los resultados aportados por Schwartz, Neale, Marco, Shiffman y Stone (1999) indicaron que los estilos de afrontamiento apenas explicaban más allá del $3 \%$ de la varianza de las estrategias de afrontamiento.

La controversia de estos resultados plantea la necesidad de continuar examinando en qué medida la tendencia a afrontar el estrés de un modo consistente, influye sobre las estrategias de afrontamiento activadas ante un estresor concreto. Hay que señalar también que la dimensión referida al nivel de especificidad vs. generalidad ha generado puntos de vista teóricos diferentes respecto de la valoración secundaria.

Bajo la óptica de la teoría sociocognitiva (Bandura, 1997), la percepción de autoeficacia, definida como la creencia personal de ser capaz de llevar a cabo un curso de acción concreto, podría entenderse como una forma de valoración secundaria, ajustándose su evaluación a los requerimientos situacionales en los que se desenvuelve la conducta. Otros investigadores (Martín-Aragón et al., 2001), sin embargo, han sugerido una concepción de la autoeficacia con un nivel de generalización más alto. En esta línea, y dentro del dominio de la salud, han propuesto la Competencia Percibida en Salud (CPS; Smith, Wallston y Smith, 1995), definiéndola como la creencia que tiene una persona sobre su capacidad para manejar las cuestiones relativas a su salud, y poder conseguir un estado de bienestar.

Diferentes estudios realizados en el ámbito del afrontamiento y la salud (Rueda y Pérez-García, 2004a, 2004b, Sanjuán, Pérez-García y Bermúdez, 2000) han puesto de relieve que, tanto la competencia percibida en salud como la percepción de autoeficacia específica, fomentan 
el empleo de estrategias de afrontamiento dirigidas a la solución del problema, y disminuyen el uso de las estrategias centradas en la emoción.

A pesar de estos datos, ningún estudio, hasta el momento, ha abordado la forma cómo la CPS podría estar afectando tanto los procesos de valoración, como las estrategias de afrontamiento que se inician ante un problema de salud determinado, punto éste que merece una atención más detenida.

Asimismo, en lo que respecta a la predicción de las estrategias de afrontamiento, la valoración primaria y la secundaria han demostrado ser determinantes principales. En este sentido diferentes trabajos (Chang, 1998; Major, Richards, Cozzarelli, Cooper y Zubeck, 1998; Tobón, Vinaccia y Sandín, 2004) han mostrado que las variables de personalidad más generales apenas influían sobre las estrategias de afrontamiento utilizadas ante un suceso estresante, en comparación con el efecto que tuvieron las valoraciones.

Teniendo en cuenta esta evidencia empírica, un aspecto que ha requerido nuestra atención es la posibilidad de contrastar, con respecto a las respuestas concretas de afrontamiento, la utilidad predictiva de variables más generales, es decir los estilos de afrontamiento y la CPS, con la de otros factores más específicos, como son los procesos de valoración; estrategia ésta que permitiría obtener una visión más integral y comprehensiva del peso que tienen cada una de estas variables dentro del proceso de estrés.

\section{Objetivos e hipótesis}

A partir de la literatura revisada se disenó un estudio dirigido a estudiar la asociación entre los estilos y las estrategias de afrontamiento que se ponían en marcha ante un hipotético problema de salud.
Asimismo se trató de determinar cuál era la contribución que hacían los estilos de afrontamiento y la competencia percibida en salud, frente a la que aportaban los procesos de valoración, en relación con las respuestas concretas de afrontamiento. Por último se investigó cuál era la influencia que ejercía la competencia percibida en salud sobre los procesos de valoración y las estrategias de afrontamiento.

En consecuencia, establecimos como primera hipótesis que los estilos de afrontamiento influirían de forma moderada sobre las respuestas concretas de afrontamiento dadas ante el supuesto problema de salud. En segundo lugar, especulamos que los procesos de valoración primaria y secundaria, por estar más ligados a la situación, contribuirían, en mayor grado que los estilos de afrontamiento y la competencia percibida en salud, a explicar las estrategias concretas de afrontamiento.

Finalmente esperamos que la competencia percibida en salud se asociaría positivamente con una evaluación poco amenazante del problema, con la expectativa de ser capaz de afrontarlo con eficacia, y con el empleo de estrategias de afrontamiento centradas en la solución del problema.

\section{MÉTODO}

\section{Participantes}

La muestra estuvo comprendida por 110 estudiantes de los primeros cursos de Psicología ( $79 \%$ mujeres y $21 \%$ hombres), con una edad media de 33 años. El rango de edad estuvo comprendido entre los 18 y los 67 años. A los participantes que quisieron participar en el estudio se les entregó directamente un cuadernillo con una serie de escalas referidas a aspectos de la personalidad y la salud. Los participantes debían rellenar los cuadernillos en su domicilio, y devolverlo posteriormen- 
te por correo a la Facultad de Psicología. En total se entregaron 330 cuadernillos, de los cuales se recibieron $110(33 \%)$.

\section{Variables}

Para evaluar la forma como se valoraba y reaccionaba ante un hipotético acontecimiento estresante, se diseñó un escenario relativo a un problema de salud. Los participantes debían imaginar que, después de tomarse casualmente la tensión, se les informaba que sus presiones arteriales eran altas: $16 \mathrm{mmHg}$ la presión sistólica, y $9 \mathrm{mmHg}$ la diastólica.

Estilos de afrontamiento. Los estilos se midieron a través del Cuestionario de Afrontamiento de las situaciones estresantes (CISS; Endler y Parker, 1994, adaptación española por Sánchez-Elvira, 1997). El CISS consta de 48 ítems, con cinco puntos de respuesta y agrupados en tres subescalas: afrontamiento centrado en el problema, afrontamiento dirigido a la emoción y afrontamiento centrado en la evitación. Esta última subescala se compone, a su vez, de dos dimensiones: la primera basada en la evitación de carácter general, y la segunda centrada en el uso de recursos sociales para distraerse del problema. En nuestro estudio consideramos como estilo de evitación la suma de las puntuaciones dadas a los items incluidos en la primera subescala (evitación general), ya que esta dimensión se ha asociado con unas consecuencias más negativas (Endler y Parker, 1994). Los coeficientes alpha oscilaron entre .85 (afrontamiento referido a la evitación) y.90 (afrontamiento dirigido a la emoción).

Competencia percibida en salud. La Escala de Competencia Percibida en Salud (ECPS, Smith et al., 1995) mide la percepción que tiene la persona sobre su capacidad para resolver las cuestiones relacionadas con la salud. Este instrumento consta de 8 ítems, cuatro de los cuales se puntúan de forma invertida. El formato de respuesta es tipo Likert con cinco puntos. En nuestro estudio el coeficiente alpha de esta medida fue de 0,86.

Valoración primaria. Esta variable se midió a través de 10 items que incluían las siguientes dimensiones: un reto, perjudicial, importante, amenazante, grave, controlable, conocida, inesperada, ambigua y preocupante. El formato de respuesta fue tipo Likert de cinco puntos, oscilando entre "Nada» y "Mucho».

Valoración secundaria. Para medir la percepción de autoeficacia con respecto al manejo de la situación planteada, se emplearon 4 items mediante los cuales los participantes debían estimar en qué medida (a) se veían capaces de hacer frente a ese problema de salud; (b) eran capaces de relajarse; (c) podían pensar en otra cosa; y (d) podían hacer algo directamente para solucionar el problema. El formato de respuesta se graduó en cinco puntos (donde $1=$ Nada y $5=$ Mucho).

Estrategias de afrontamiento. Esta variable se midió a través de una escala de 9 items, con un formato de respuesta tipo Likert de cinco puntos. Los ítems se agruparon en tres categorías: afrontamiento centrado en el problema, afrontamiento centrado en el manejo negativo de la emoción, y afrontamiento dirigido al manejo positivo de la emoción. Las dos primeras dimensiones son consistentes con las incluidas en diferentes instrumentos de medición del afrontamiento (Carver et al., 1989), mientras que la última responde a la distinción teórica sugerida por algunos investigadores (Muela, et al., 2002) sobre el empleo de la regulación efectiva o inefectiva de las emociones como estrategia de afrontamiento. Los coeficientes alpha obtenidos fueron los siguientes: 0,73 para el afrontamiento dirigido al problema, 0,72 para el afrontamiento negativo de la emoción, y 0,68 para el afrontamiento positivo de la emoción. 


\section{RESULTADOS}

\section{Resultados preliminares y estadísticos descriptivos}

En primer lugar se realizaron una serie de análisis para examinar la estructura factorial de las escalas de valoración primaria, valoración secundaria y estrategias de afrontamiento incluidas en el supuesto de salud. Los items relativos a la valoración primaria fueron sometidos a un análisis factorial de componentes principales usando rotación varimax. Los resultados obtenidos pusieron de manifiesto dos factores interpretables que daban cuenta de un $52 \%$ de la varianza. En el primer factor, llamado "percepción de amenaza", saturaron cinco items ("perjudicial», "importante», "amenazante», "grave» y "preocupante») con un peso factorial superior a 0,70 (coeficiente alpha $=0,88$ ). En el segundo factor, denominado "percepción de reto", saturaron los dos items relativos al control y el reto, siendo sus cargas factoriales superiores a 0,70 (coeficiente alpha $=0,57$ ). Los items relativos a "una situación conocida», "inesperada» $y$ "ambigua» fueron eliminados puesto que no saturaron claramente en ninguno de los dos factores definidos.

Un segundo análisis factorial se realizó con los ítems pertenecientes a la escala de valoración secundaria. En este caso se utilizó una rotación oblicua dada la interrelación entre las variables. El análisis mostró un único factor, que daba cuenta del $52 \%$ de la varianza y en el que los pesos factoriales de los items saturaron por encima de 0,30 . Teniendo en cuenta esta estructura unidimensional, las puntuaciones dadas a los cuatro items se sumaron para obtener un índice de "percepción de autoeficacia respecto del afrontamiento" (coeficiente alpha $=0,68$ ).

Finalmente los nueve ítems referidos a las estrategias de afrontamiento fueron también sometidos a un análisis factorial exploratorio, mostrando una solución trifactorial que explicaba un $67 \%$ de la varianza. En el primer factor saturaron los ítems incluidos en el afrontamiento "centrado en el problema«, en el segundo los referidos al "manejo negativo de la emoción «, y en el tercero los relativos al "manejo positivo de la emoción «. Los pesos factoriales de casi todos los items fueron superiores a 0,70. Un ítem de la subescala del manejo positivo de la emoción mostró una carga factorial superior a 0,30 en dos de los factores. Por este motivo, no se tuvo en cuenta dicho ítem en los análisis subsiguientes.

Los estadísticos descriptivos (medias, desviaciones estándar y rango de respuestas) de las variables utilizadas se muestran en la Tabla 1.

Tabla 1. Medias, desviaciones típicas y rango de las variables medidas

\begin{tabular}{lrrrr}
\hline & \multicolumn{1}{c}{ M } & \multicolumn{1}{c}{ DT } & Rango real & Rango posible \\
\hline Estilo Afrontamiento Problema & 62,10 & $\mathbf{8 , 2 5}$ & $\mathbf{3 6 - 8 0}$ & $\mathbf{1 6 - 8 0}$ \\
Estilo de Afrontamiento Emoción & 44,21 & 11,97 & $16-70$ & $16-80$ \\
Estilo de Afrontamiento Evitación & 19,50 & 7,27 & $8-38$ & $8-40$ \\
Competencia Percibida Salud & 31,16 & 4,15 & $20-40$ & $8-40$ \\
Valoración Amenaza & 15,17 & 3,78 & $5-25$ & $5-25$ \\
Valoración Reto & 7,69 & 1,50 & $4-10$ & $2-10$ \\
Percepción Autoeficacia & 15,49 & 2,38 & $11-20$ & $4-20$ \\
Estrategias Afrontamiento Problema & 11,38 & 2,58 & $4-15$ & $3-15$ \\
Estrategias Afrontamiento Emocional Positivo & 5,59 & 2,02 & $2-10$ & $2-10$ \\
Estrategias Afrontamiento Emocional Negativo & 6,56 & 2,20 & $3-13$ & $3-15$ \\
\hline
\end{tabular}


Relaciones entre los estilos y las estrategias de afrontamiento

Para determinar la asociación entre los estilos y las estrategias de afrontamiento, se hallaron las correlaciones entre estos dos tipos de variables. Como se aprecia en la Tabla 2, el estilo de afrontamiento centrado en el problema correlacionó de forma moderada y positiva con las estrategias de afrontamiento referidas a la misma dimensión, así como con las estrategias orientadas al manejo positivo de la emoción. No obstante, su conexión con las estrategias dirigidas al manejo negativo de la emoción no fue significativa. Los estilos de afrontamiento dirigidos a la emoción y a la evitación también correlacionaron positiva y moderadamente con las estrategias focalizadas en el manejo negativo de la emoción, aunque no se relacionaron significativamente con las estrategias centradas en el manejo positivo de la emoción. De estos dos estilos, sólo el orientado a la evitación se asoció positivamente con las estrategias de afrontamiento dirigidas a la solución del problema.

Para determinar si la asociación entre los estilos y las estrategias de afrontamiento estaba influida por una tercera variable (la CPS -competencia percibida en salud-o los procesos de valoración), se obtuvieron las correlaciones parciales entre los dos tipos de afrontamiento, controlando el posible efecto de estos factores. Los resultados indicaron que las relaciones del estilo de afrontamiento centrado en el problema y del orientado a la evitación con las estrategias dirigidas al problema, fueron no significativas en ambos casos. Lo mismo sucedió con la asociación entre el afrontamiento orientado a la evitación y las estrategias dirigidas al manejo negativo de la emoción. En contraste, la correlación entre el estilo de afrontamiento centrado en la emoción y las estrategias dirigidas al manejo inefectivo de la emoción, sólo disminuyó ligeramente $(r=0,25 ; p<0,01)$. De esta forma, parece que la CPS y los procesos específicos de valoración estarían modulando las conexiones del estilo de afrontamiento orientado al problema y el de evitación con las respuestas situacionales de afrontamiento, siendo menor su efecto cuando el estilo de afrontamiento se refiere a aspectos emocionales.

\section{CPS, los procesos de valoración y estrategias de afrontamiento}

A continuación se llevó a cabo un análisis correlacional con la CPS, los procesos de valoración y las estrategias de afrontamiento. La inspección de la Tabla 3 pone de manifiesto que, de acuerdo con la hipótesis planteada, la CPS amortiguaba la valoración amenazante del problema de salud y promovía una mayor sensación de confianza en la capacidad personal para hacerle frente. Además, la CPS tendía a facilitar el empleo de estrategias dirigidas al problema y paliaba aquellas otras relacionadas con el manejo negativo de la emoción.

Tabla 2. Correlaciones entre los estilos y las estrategias de afrontamiento

\begin{tabular}{|c|c|c|c|}
\hline & $\begin{array}{c}\text { Estilo } \\
\text { Afrontamiento } \\
\text { Problema } \\
\end{array}$ & $\begin{array}{c}\text { Estilo } \\
\text { Afrontamiento } \\
\text { Emoción }\end{array}$ & $\begin{array}{c}\text { Estilo } \\
\text { Afrontamiento } \\
\text { Evitación } \\
\end{array}$ \\
\hline Estrategias Afrontamiento Problema & $0,31^{\star *}$ & $-0,08$ & 0,27 ** \\
\hline Estrategias Afrontamiento Emocional Positivo & $0,28^{* *}$ & $-0,15$ & 0,16 \\
\hline Estrategias Afrontamiento Emocional Negativo & $-0,03$ & $0,29^{\star \star}$ & $0,21 *$ \\
\hline
\end{tabular}

${ }^{\star \star} \mathrm{p}<0,01 ;{ }^{*} \mathrm{p}<0,05$. 
Tabla 3. Correlaciones de la CPS (Competencia Percibida en Salud) con las valoración y las estrategias de afrontamiento

\begin{tabular}{lc}
\hline & CPS \\
\hline Percepción Amenaza (Valoración Primaria) & $-0,19^{\star}$ \\
Percepción Reto (Valoración Primaria) & 0,13 \\
Percepción Autoeficacia (Valoración Secundaria) & $0,50^{\star \star \star}$ \\
Estrategias de Afrontamiento Problema & $0,29^{\star \star}$ \\
Estrategias de Afrontamiento Emocional Positivo & 0,10 \\
Estrategias de Afrontamiento Emocional Negativo & $-0,22^{\star}$ \\
\hline
\end{tabular}

${ }^{* \star *} \mathrm{p}<0,001 ;{ }^{* *} \mathrm{p}<0,01 ;{ }^{*} \mathbf{p}<0,05$

Predicción de las estrategias de afrontamiento a partir de los estilos, la CPS y las valoraciones

Finalmente se realizaron una serie de análisis jerárquicos para determinar la contribución de los estilos de afrontamiento y la CPS, así como la de las valoraciones, con respecto a las estrategias de afrontamiento. Atendiendo a su nivel de generalidad, en el primer paso se introdujeron los estilos de afrontamiento y la CPS, y en el segundo los procesos de valoración. Con respecto a las estrategias orientadas al problema (ver Tabla 4), y en contraste con lo esperado, los estilos de afrontamiento y la CPS explicaron el mismo porcentaje de varianza $\left(R^{2}=0,17\right)$ que los procesos de valoración. Más específicamente, el hecho de sentirse competente en el manejo de la salud y de valorar el supuesto problema como una amenaza instaban a utilizar un tipo de estrategias centradas en la solución del problema. Los estilos de afrontamiento, por su par- te, no predijeron ninguna de estas respuestas de afrontamiento.

En cuanto a las estrategias de afrontamiento centradas en el manejo positivo de la emoción (ver Tabla 5), los estilos y la competencia explicaron un porcentaje de variabilidad $\left(R^{2}=0,10\right)$ muy similar, aunque ligeramente superior, al aportado por las valoraciones $\left(\Delta R^{2}=0,08\right)$. En concreto, el estilo de afrontamiento centrado en el problema predijo positivamente este criterio, mientras que la valoración de amenaza presentó un peso negativo.

En el último análisis de regresión se utilizaron como criterio las estrategias de afrontamiento dirigidas al manejo negativo de la emoción (ver Tabla 6). En este caso los procesos de valoración fueron los que explicaron el porcentaje más alto de la variabilidad de esta forma situacional de afrontamiento $\left(\Delta R^{2}=0,33\right)$, por encima de los estilos y la CPS $\left(R^{2}=0,11\right)$. Asimismo, sólo la valoración de amenaza y la percepción de autoeficacia emergieron como predictores significativos,

Tabla 4. Regresión Jerárquica prediciendo las estrategias de afrontamiento orientadas al problema

\begin{tabular}{|c|c|c|c|c|c|}
\hline & $\beta$ & $\mathrm{R}^{2}$ & $\Delta \mathbf{R}^{2}$ & F & g.l. \\
\hline Estilo Afrontamiento - Problema & 0,04 & 0,17 & 0,17 & $5,53^{* \star *}$ & 4,109 \\
\hline Estilo Afrontamiento - Emoción & 0,03 & & & & \\
\hline Estilo Afrontamiento - Evitación & 0,17 & & & & \\
\hline CPS & $0,28^{*}$ & & & & \\
\hline Valoración Amenaza & $0,41 * * *$ & 0,34 & 0,17 & $7,50 * \star *$ & 7,109 \\
\hline Valoración Reto & $-0,04$ & & & & \\
\hline Percepción Autoeficacia & 0,18 & & & & \\
\hline
\end{tabular}

${ }^{* * *} \mathrm{p}<0,001 ;{ }^{* *} \mathrm{p}<0,01 ;{ }^{*} \mathrm{p}<0,05$. 
Tabla 5. Regresión jerárquica prediciendo las estrategias de afrontamiento de manejo positivo de la emoción

\begin{tabular}{lccccc}
\hline & $\beta$ & $\mathrm{R}^{2}$ & $\Delta \mathrm{R}^{2}$ & $\mathrm{~F}$ & $\mathrm{~g} .1$. \\
\hline Estilo Afrontamiento - Problema & $0,24^{\star}$ & 0,10 & 0,10 & $2,94^{\star}$ & 4,109 \\
Estilo Afrontamiento - Emoción & $-0,15$ & & & & \\
Estilo Afrontamiento - Evitación & 0,17 & & & & \\
CPS & $-0,19^{\star}$ & & & & \\
\hline Valoración Amenaza & $-0,19^{\star}$ & 0,18 & 0,08 & $3,36^{\star \star}$ & 7,109 \\
Valoración Reto & 0,16 & & & & \\
Percepción Autoeficacia & 0,18 & & & & \\
\hline
\end{tabular}

${ }^{*} \mathrm{p}<0,01 ;{ }^{*} \mathrm{p}<0,05$

siendo el coeficiente beta de la amenaza positivo, y el de la percepción de autoeficacia negativo.

\section{DISCUSIÓN}

En este estudio nos planteamos examinar, en primer lugar, los efectos del afrontamiento disposicional sobre las estrategias de afrontamiento iniciadas ante un supuesto problema de salud. En este sentido encontramos que la influencia ejercida por el afrontamiento disposicional era relativamente moderada. En concreto, el estilo de afrontamiento centrado en el problema correlacionó positivamente con las estrategias de afrontamiento orientadas al problema y con las dirigidas al manejo positivo de la emoción, relación ésta última que, además, se mantuvo en el análisis multivariado. Los estilos de afrontamiento orientados a la emoción y a la evitación, por su parte, se asociaron de forma moderada con las estrategias dirigidas al manejo negativo de la emoción, aunque en la ecuación de regresión sólo el estilo centrado en la emoción mantuvo un peso significativo. En este caso obtuvimos también una relación directa entre el afrontamiento disposicional y el situacional, ya que, tras eliminar el efecto de la posibles CPS y los procesos de valoración, el estilo focalizado en la emoción continuó asociándose de forma significativa con sus respectivas estrategias de afrontamiento.

En conjunto, estas relaciones son consistentes con las evidenciadas por otros estudios (Carver y Scheier, 1994; Carver et al., 1989). Endler, Parker y Summerfeldt (1998), por ejemplo, utilizando el CISS como medida de afrontamiento diposicional y situacional, encontraron una fuerte asociación entre los dos tipos de afrontamiento cuando la dimensión empleada se refería a la emoción, siendo esta conexión bastante más baja cuando las formas de afrontamiento se orientaban al problema.

Tabla 6. Regresión jerárquica prediciendo las estrategias de afrontamiento de manejo negativo de la emoción

\begin{tabular}{|c|c|c|c|c|c|}
\hline & $\beta$ & $\mathbf{R}^{2}$ & $\Delta \mathrm{R}^{2}$ & $\mathrm{~F}$ & g.l. \\
\hline Estilo Afrontamiento - Problema & 0,02 & 0,11 & 0,11 & $3,48^{*}$ & 4,109 \\
\hline Estilo Afrontamiento - Emoción & $0,21^{\star}$ & & & & \\
\hline Estilo Afrontamiento - Evitación & 0,07 & & & & \\
\hline CPS & 16 & & & & \\
\hline Valoración Amenaza & $0,51^{\star \star \star}$ & 0,44 & 0,33 & $11,68^{\star \star \star}$ & 7,109 \\
\hline Valoración Reto & $-0,02$ & & & & \\
\hline Percepción Autoeficacia & $-0,36^{\star \star \star *}$ & & & & \\
\hline
\end{tabular}

${ }^{\star \star *} \mathrm{p}<0,001 ;{ }^{* *} \mathrm{p}<0,01 ;{ }^{*} \mathrm{p}<0,05$. 
Estos datos vendrían a sugerir que el afrontamiento situacional orientado a la solución del problema podría estar más influido por factores contextuales, y por tanto cambiar según las demandas de la situación, mientras que el afrontamiento emocional estaría determinado por aspectos personales, adoptando así un carácter más estable.

Asimismo, los distintos pesos que mostraron el estilo de afrontamiento centrado en el problema y la valoración de amenaza en la predicción de las estrategias orientadas al manejo constructivo de la emoción, estarían indicando que el empleo de este estilo podría estar contribuyendo a alterar la situación de estrés haciéndola menos amenazante, además de dar lugar a un estado emocional más reconfortante y positivo.

Nuestro estudio también investigó la asociación de la competencia con las valoraciones y las estrategias de afrontamiento originadas en torno al problema de salud. En consonancia con la hipótesis planteada, la competencia promovió la creencia de ser capaz de manejar la situación planteada, y el empleo de acciones de afrontamiento dirigidas a solucionarla, al mismo tiempo que disminuía la valoración amenazante del problema.

Estos resultados, no sólo replican los hallados en investigaciones previas (Rueda y Pérez-García $2004_{a}, 2004_{b}$ ), sino que también intentan ir más allá del enfoque interindividual, examinando el funcionamiento de esta expectativa dentro del contexto específico donde se genera el estrés.

Por último, en cuanto a la utilidad predictiva de los estilos de afrontamiento y la CPS, en contraste con la de los procesos de valoración, los resultados confirmaron parcialmente la hipotesis establecida. Los estilos y la CPS dieron cuenta de un porcentaje de variabilidad similar al aportado por las valoraciones, en lo que respecta a las estrategias de afrontamiento focalizadas en el problema y el manejo positivo de la emoción. Sólo en relación con las estrategias orientadas al manejo negativo de la emoción, los procesos de valoración explicaron un mayor porcentaje de varianza.

Una explicación plausible de la preponderancia de los estilos de afrontamiento y la competencia podría apoyarse en el hecho de que el supuesto diagnóstico de una presión arterial alta podría constituir una situación nueva para los participantes del estudio, circunstancia ésta en la que se ha constatado que las variables de personalidad más generalizadas tienen una mayor relevancia (Folkman, 1984).

En consonancia con esta idea se podría entender el efecto que tuvo la CPS sobre las estrategias de afrontamiento centrades en el problema, efecto que llegó a ser más importante que el de la percepción de autoeficacia. Este dato podría sugerir que, ante la presentación de una información amenazante, como era el conocimiento de una presión arterial elevada, el inicio de acciones dirigidas a solucionar el problema estaría influenciado por la competencia percibida relativa al manejo de la salud, antes que por las creencias específicas de autoeficacia. Esta conclusión es, no obstante, tentativa, y requiere de futuras investigaciones en las que se estudie el funcionamiento de ambas expectativas en una situación real de hipertensión.

De otro lado, el hecho de que las valoraciones fueran unos predictores importantes en relación con las estrategias de afrontamiento emocional negativo, es congruente con la literatura revisada (Chang, 1998; Major et al., 1998; Tobón et al., 2004). A este respecto, Terry (1994), en su estudio sobre la influencia del afrontamiento disposicional y las variables personales sobre el afrontamiento situacional, observó que la valoración primaria y secundaria eran los principales predictores de las estrategias centradas en la culpa y el escape. 
Finalmente resulta llamativo el papel que en nuestro estudio desempeñó la valoración de amenaza. Este tipo de percepción se asoció con un mayor uso, tanto de las estrategias de afrontamiento centradas en el manejo negativo de la emoción, como de las orientadas a la solución del problema. Este dato daría a entender que, por una parte, la percepción de amenaza, cuando se acompaña de un estilo de afrontamiento centrado en la emoción, da lugar a una serie de respuestas encaminadas a reducir el malestar emocional. Por otra parte, este tipo de valoración también puede originar un proceso constructivo, el cual, admitiendo la existencia de un riesgo potencial, no llega a bloquear la capacidad de afrontamiento de la persona permitiéndole manejar la resolución del estrés.

Como posibles limitaciones, habría que señalar que el estresor elegido para examinar las valoraciones y las estrategias de afrontamiento, fue un hipotético problema de salud. Esta cuestión implica que los datos presentados en esta investigación deberían compararse con los obtenidos en un escenario real, donde se hubiera realizado un diagnóstico de hipertensión. Además, para poder generalizar estos resultados, sería conveniente analizar la influencia de las variables elegidas con respecto a diferentes problemas de salud. Este enfoque facilitaría examinar la consistencia transituacional de los estilos de afrontamiento y la CPS, y su posible variación en consonancia con los procesos de valoración y con las propias características del estresor.

En conclusión, este estudio apoya la idea de que los estilos de afrontamiento influyen de forma moderada sobre el afrontamiento situacional. También amplía el horizonte de que se dispone sobre las relaciones entre la CPS y los procesos de valoración y afrontamiento más contextuales. Finalmente la investigación pone de manifiesto que las variables de personalidad más generales contribuyen, en igual medida que los procesos de valoración, a explicar determinadas acciones de afrontamiento, aunque el peso de estos procesos destaca en el afrontamiento focalizado en la emoción negativa.

\section{REFERENCIAS}

Bandura, A. (1997). Self-efficacy: The exercise of control. New York: W.H. Freeman and Company.

Bouchard, G., Guillemette, A., y LandryLéger, N. (2004). Situational and dispositional coping: An examination of their relation to personality, cognitive appraisals, and psychological distress. European Journal of Personality, 18, 221-238.

Carver, C.S., y Scheier, M.F. (1994). Situational coping and coping dispositions in a stressful transaction. Journal of Personality and Social Psychology, 66, 184-195.

Carver, C.S., Scheier, M.F., y Weintraub, J.K. (1989). Assessing coping strategies: A theoretically based approach. Journal of Personality and Social Psychology, 56, 267-283.

Chang, E.C. (1998). Dispositional optimism and primary and secondary appraisal of a stressor: Controlling for confounding influences and relations to coping and psychological and physical adjustment. Journal of Personality and Social Psychology, 74, 1109-1120.

Endler, N. S., y Parker, J. D. A. (1994). Assessment of multidimensional coping: Task, emotion, and avoidance strategies. Psychological Assessment, 6, 50-60.

Endler, N.S., Parker, J.D.A., y Summerfeldt, L.J. (1998). Coping with health problems: Developing a reliable and valid multidimensional measure. Psychological Assessment, 10, 195-205.

Folkman, S. (1984). Personal control and stress and coping processes. A theoretical analysis. Journal of Personality and Social Psychology, 46, 839-852.

Folkman, S., Lazarus, R.S., Dunkel-Schetter, C., DeLongis, A., y Gruen, R.J. (1986). 
Dynamics of a stressful encounter : cognitive appraisal, coping, and encounter outcomes. Journal of Personality and Social Psychology, 50, 992-1003.

Lazarus, R.S., y Folkman, S. (1987). Transactional theory and research on emotions and coping. European Journal of Personality, 1, 141-169.

Major, B., Richards, C., Cozzarelli, C., Cooper, M.L., y Zubeck, J. (1998). Personal resilience, cognitive appraisals, and coping: an integrative model of adjustment to abortion. Journal of Personality and Social Psychology, 74, 735-752.

Martín-Aragón, M., Pastor, M.A., Lledó, S., López-Roig, M.C., Terol, M.C., y Rodríguez-Marín, J. (2001). Percepción de control en el síndrome fibromiálgico: variables relacionadas. Psicothema, 13, 586-591.

Muela, J.A., Torres, C.J.,y Peláez, E. (2002). Comparación entre distintas clasificaciones de las estrategias de afrontamiento en cuidadores de enfermos de Alzheimer. $P$ sicothema, 14, 558-563.

Rueda, B., y Pérez-García, A.M. (2004a). Análisis comparativo de la competencia percibida general y la específica de salud. Ansiedad y Estrés, 10, 127-139.
Rueda, B., y Pérez-García, A.M. (2004b). Personalidad y percepción de autoeficacia: Influencia sobre el bienestar y el afrontamiento de los problemas de salud. Revista de Psicopatología y Psicología Clínica, 9, 205-219

Sánchez-Elvira, A. (1997). Spanish Coping Inventory for Stressful Situations (CISS. Endler \& Parker, 1990). Canada: MultiHealth Systems Inc.

Sanjuán, P., Pérez-García, A.M., y Bermúdez, J. (2000). Escala de autoeficacia general: datos psicométricos de la adaptación para población española. Psicothema, 12, 509513.

Smith, M.S., Wallston, K.A., y Smith, C.A. (1995). The development and validation of the Perceived Health Competence Scale. Health Education Research, 10, 51-64.

Terry, D.J. (1994). Determinants of coping: the role of stable and situational factors. Journal of Personality and Social Psychology, $66,895-910$.

Tobón, S., Vinaccia, S., y Sandín, B. (2004). Modelo procesual del estrés en la dispepsia funcional: Implicaciones para la evaluación y el tratamiento. Revista de Psicopatología y Psicología Clínica, 9, 81-98. 